\title{
La Importancia de la Investigación en el Proyecto Educativo Institucional ${ }^{1}$

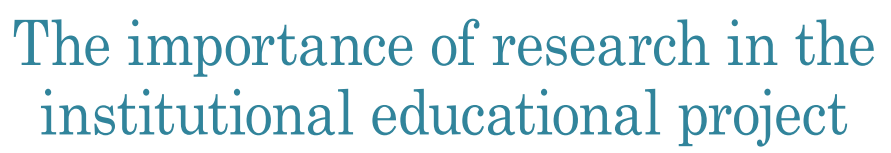

DOI: http://dx.doi.org/10.17981/cultedusoc.9.2.2018.02

Fecha de recepción: 10/06/2018. Fecha de aceptación: 05/09/2018

Leovigildo Sierra-Mora ${ }^{2}$

Yohenis López-Flórez; Camilo Castro-Castro; Melba Flórez-Quiroz; Celina Flórez-Sierra;

Luis Ferreira-Alfaro; Alexi Tinoco-Flórez; Ada Luz Martínez-Hernández;

Emiro Lemus-Pacheco; Yesid Barros-Cantillo; Yanet Fonseca-Guerra;

Nidia Flórez-de Miranda; Carmen González-Rodríguez; Rocío Hernández-Hernández ${ }^{3}$

Para citar este artículo

Sierra-Mora, L., López-Flórez, Y., Castro-Castro, C., Flórez-Quiroz, M., Flórez-Sierra, C., Ferreira-Alfaro, L., Tinoco-Flórez, A., Martínez-Hernández, A., Lemus-Pacheco, E., Barros-Cantillo, Y., Fonseca-Guerra, Y., Flórez-de Miranda, N., González-Rodríguez, C. y Hernández-Hernández, R. (2018). La Importancia de la Investigación en el Proyecto Educativo Institucional. Cultura. Educación y Sociedad 9(2), 21-31. DOI: http://dx.doi. org/10.17981/cultedusoc.9.2.2018.02

\section{Resumen}

El presente artículo se enfoca en el proyecto educativo, que de manera flexible permita la articulación de la investigación con el diseño curricular de la IEDR de Ricaurte. Los referentes teóricos de Duque (2016), Mejía (2013), Cuevas et. al. (2016), proponen que la investigación ejerce un impacto vinculante entre la práctica pedagógica y la teoría, brindándoles a los estudiantes la posibilidad de apropiarse de la lógica del conocimiento y de herramientas propias de la ciencia. Se asume un abordaje cualitativo, se tomó como muestra (40) estudiantes de diferentes grados. Dentro de los hallazgos encontrados, existe gran afinidad entre los estudiantes y las actividades investigativas, lo cual indica que el proyecto tiene un alto nivel de aceptación, sin embargo, se hace necesaria la resignificación del PEI para obtener resultados significativos.

Palabras clave: Proyecto educativo, Investigación Científica, Aprendizaje basado en Proyectos, diseño curricular, conocimiento.

\begin{abstract}
This article focuses on the educational project, which in a flexible way allows the articulation of the research with the curricular design of the IEDR of Ricaurte. The theoretical references of Duque (2016), Mejia (2013), Cuevas et. to the. (2016), propose that research exerts a binding impact between pedagogical practice and theory, giving students the possibility of appropriating the logic of knowledge and tools of science. A qualitative approach is assumed; 40 students of different grades were taken as sample. Among the findings found, there is great affinity between the students and the research activities, which indicates that the project has a high level of acceptance, however, it is necessary to resignify the PEI to obtain significant results.
\end{abstract}

Keywords: Educational project, Scientific Research, Project-based Learning, desing curricular, knowledge.

\footnotetext{
${ }^{1}$ Este artículo ha sido derivado del Programa de Fortalecimiento de la Cultura Ciudadana y Democrática CT+I a través de la IEP apoyada en TIC en el Departamento de Magdalena: CICLON.

${ }^{2}$ Líder del grupo de investigación "Vigias de la ciénaga la Rinconada". Correspondencia: Email: leovigildosierramora@gmail.com

${ }^{3}$ Docentes pertenecientes al grupo de investigación "Vigias de la ciénaga la Rinconada" de la IED Hato Viejo y Ricaurte. Ciclón Magdalena, estrategia $\mathrm{N}^{\circ} 2$.
}

- The author; licensee Universidad de la Costa - CUC. 


\section{Introducción}

En todos los niveles de educación, en especial los niveles primarios, es necesaria la integración de la investigación científica apoyada en las TIC en el diseño curricular.

La investigación debe ser un elemento integrado al currículo, y para esto, deben existir condiciones básicas que lo permitan. Los cuestionamientos que de manera constante sufre el sistema educativo, y la agitada dinámica social que demanda cambios urgentes en nuestras instituciones para dar respuestas a las problemáticas que amenazan a las comunidades, son un excelente caldo de cultivo la investigación en la estructura curricular de las instituciones educativas desde los primeros grados de enseñanza.

Es fundamental la coexistencia de factores que permitan llevar a cabo un diseño curricular innovador que incluya los proyectos de investigación como ejes transversales que dinamicen los planes de estudios en la institución. Según Castro (2017) La gestión administrativa debe ser visionaria, dinámica y muy emprendedora para conseguir y proveer a la institución del equipamiento necesario para desarrollar los procesos de manera eficaz.

La labor pedagógica ha de estar acorde a las circunstancias, con docentes comprometidos y preparados para asumir los retos para conducir los procesos de enseñanza de forma constructiva. La dimensión tecnológica está relacionada con las herramientas que permitan el acceso a la información localizadas en las redes de comunicación que proporcionan a los estudiantes y docentes las posibilidades de construir conocimientos, y adquirir saberes específicos de acuerdo al área de interés.
Lo anterior es posible si los miembros de la comunidad educativa, emprenden la delicada labor de reestructurar el currículo de la institución ubicando en el sitio estratégico a la investigación científica apoyada en las TIC de acuerdo a los lineamientos del programa Ciclón, por lo tanto, el objetivo de la presente investigación es Integrar al PEI de la I.E.D de Ricaurte los lineamientos de la IEP apoyada en las TIC.

El currículo de la I.E.D. de RicaurteGuamal, Magdalena, carece de criterios que permitan la integración de los lineamientos de la I. E. P. apoyada en TIC del programa Ciclón, que han orientado el proyecto de investigación acerca del mejoramiento del medio ambiente con relación a la ciénaga la Rinconada, liderados por los estudiantes y docentes en pro de la solución de las problemáticas que aquejan a la comunidad.

Si bien, en la institución se vienen desarrollando proyectos de investigación que responden a necesidades puntuales de la comunidad que la circunda, sus contenidos no se encuentran integrados al PEI de la institución, ya que en los diferentes elementos que lo conforman no está incluida la actividad investigativa de carácter científico apoyada en las TIC que integre saberes y conocimientos de las diversas asignaturas que se imparten en la institución.

En el actual currículo no existen criterios definidos que permitan regular o acondicionar la I.E.P al quehacer diario del docente en el aula; así mismo, el énfasis de la institución está enfocado al procesamiento de frutas y verduras y carece de pautas definidas que posibiliten implementar la investigación como estrategia pedagógica apoyada en las TIC y los diferentes proyectos están enfocados solamente a la realización 
de consultas de interés personal que poco o nada requieren de la investigación desestimando la motivación en sus procesos de ejecución. En relación al aspecto de cualificación docente, las prácticas pedagógicas carecen de estrategias que dinamicen un proceso coherente acorde con la investigación apoyada en las TIC.

Cuevas, et al. (2016), afirman que "la enseñanza de la investigación a niños desde sus primeros años de educación representa una oportunidad para fomentar el desarrollo de sus potencialidades, en particular las relacionadas con la recolección, análisis e interpretación de la información, así como la búsqueda de respuestas a problemas derivados de las asignaturas señaladas como parte de su plan académico. De ello surge la necesidad de considerar la generación de capacidades y habilidades investigativas en el marco de la planeación académica de cada materia impartida." Lo anterior evidencia la importancia de llevar a cabo un proyecto que tenga como objetivo potencializar y desarrollar, habilidades y destrezas en la primera infancia de las personas, las cuales puedan brindar facilidad al momento de afrontar ciertas situaciones que requieran de análisis y capacidades investigativas y asi darle una solución adecuada a las mismas.

Mediante amplias investigaciones se ha clarificado la importancia de las actividades prácticas en la búsqueda del conocimiento. Las actividades prácticas influyen particularmente en las competencias de ciencias, impactan de manera positiva en el interés de los estudiantes por aprender los temas a que se pretenden enseñar. Blanco (2005), destaca que la "igualdad de oportunidades ha de empezar desde el nacimiento para superar o reducir lo más tempranamente posible las situaciones de desigualdad o vulnerabilidad en las que se encuentran millones de niños y niñas en el mundo. La inversión en programas de educación y cuidado de la primera infancia puede contribuir de forma importante a una mayor igualdad social y, por tanto, al desarrollo humano." La equidad en temas de educación desde la primera edad, toma gran importancia de acuerdo con las limitadas oportunidades educativas para las personas de escasos recursos, las cuales no siempre pueden contar con un óptimo desarrollo educativo.

A pesar de los esfuerzos realizados por aumentar la cobertura y focalizar la atención en las zonas rurales y en los grupos de mayor vulnerabilidad.

Según Guerrero (2015) "existe un problema importante de desigualdad en el acceso y en la calidad de los programas que se ofrecen a los colectivos en situación de mayor vulnerabilidad. En efecto, aunque no se dispone de información suficiente y desagregada, es posible afirmar que actualmente la educación de la primera infancia no llega a quienes más la necesitan para superar su situación de desigualdad. Tampoco se dispone de información suficiente sobre la calidad de los programas que se dirigen a estos grupos, por lo que no es posible determinar si los programas existentes logran el objetivo de superar la situación de desventaja de estos niños y niñas, ni si favorecen su plena participación, desarrollo y aprendizaje." La primera infancia es la etapa más importante del ser humano, por ello se debe trabajar continuamente en maximizar las oportunidades educativas para aquellos niños y niñas que están ubicados en lugares rulares y ejercer un control para que la 
educación sea permanentemente de calidad, efectuar medidas que garanticen la permanencia y continuidad de la misma, para posteriormente obtener resultados positivos.

Para la I.E.D. de Ricaurte es de suma importancia integrar los procesos de investigación científica que desarrollan los docentes y estudiantes al PEI, realizando una estructuración que permita introducir los lineamientos de la IEP apoyada en TIC y elevar los contenidos de los proyectos a la categoría de ejes transversales.

El PEI de la I.E.D. de Ricaurte, está orientado hacia la el procesamiento de frutas y verduras con fines de desarrollar en los jóvenes una mentalidad emprendedora capaces de crear a futuro microempresas que impacten en su comunidad y la región; en la actualidad los docentes y estudiantes desarrollan el proyecto de investigación "reforestación con mangles criollos de las orillas de la ciénaga la rinconada" que corresponde a las actividades más importantes del programa de capacitación "fortalecimiento de la cultura ciudadana y democrática en CT+I a través de la IEP apoyada en TIC en el departamento del magdalena," del proyecto Ciclón, en el cual participa como comunidad educativa beneficiada, e interesada en mejorar la calidad educativa de la institución y contribuir al desarrollo de la región.

En virtud de lo anterior la articulación de los lineamientos de la IEP apoyada en TIC al diseño curricular de la institución es de vital importancia y para ello es necesario reflexionar sobre las prácticas docentes que actualmente se llevan en la I.E.D. de Ricaurte y con base a ellas establecer criterios que permitan incorporar a manera de eje transversal, que, permeando las diferentes áreas, los lineamientos de la IEP apoyada en TIC.

A través del documento, "El marco legal del currículo en Colombia”, el Ministerio de Educación Nacional emiten las normas que regulan y dan pautas para el diseño del currículo en las diferentes instituciones educativas y definiendo cada uno de sus componentes, la función que desempeñan y la manera como se articulan en el entramado curricular.

Las normas que rigen el diseño curricular son: Ley General de Educación, Ley 115 de 1994, Decreto 1860 de 1994, Decreto 1290 de 2009, Lineamientos curriculares de las diferentes áreas y los Estándares básicos de competencias en diferentes áreas.

En su artículo 76, la ley general de educación define el término currículo como "el conjunto de criterios, planes de estudio, programas, metodologías y procesos que contribuyen a la formación integral y a la construcción de la identidad cultural nacional, regional y local, incluyendo también los recursos humanos. Académicos y físicos para poner en práctica las políticas y llevar a cabo el PEI".

En relación a los criterios para la elaboración del currículo, en el decreto 1860 de 1994 se señala la importancia de actualizar permanentemente el currículo para enfocar el que hacer académico y de una manera reflexiva lograr su fácil adaptación a culturas con características determinadas, las cuales son regidas por su entorno

En concordancia con lo anterior, en el artículo 78 de la Ley General de Educación, se afirma que las instituciones mantendrán actividades de desarrollo curricular que comprendan la investigación, el diseño y la evaluación permanente del currículo; es decir, se ubica la 
investigación como un factor clave en el desarrollo de un currículo innovador que ofrece las herramientas a la comunidad educativa para que encuentre soluciones a las problemáticas del entorno más inmediato. De esta manera, el proyecto de integración curricular que emprenden los docentes en la institución responde a lo indicado por el ministerio de educación para lograr los índices de calidad que merecen todo el centro educativo del país.

Dentro del concepto de flexibilidad curricular, surge opción importante que pretende la integridad del currículo, y es la transversalidad. En el ámbito educativo, entonces, la transversalidad se refiere a una estrategia educativa en la cual algunos factores tienen mayor importancia en la formación de los estudiantes, los cuales deben estar en todos los programas, proyectos, actividades y planes de estudios contemplados en el PEI de una Institución Educativa", La transversalidad curricular implica como lo indica Fernández (2003:5) quien plantea "la utilización de nuevas estrategias, metodología y necesariamente formas de organización de los contenidos", de igual manera, Velásquez Sarria J, A, 2009 Pag 38, plantea la transversalidad como una constitución de líneas que cruzan todas las disciplinas y se rigen en elementos vertebrador del aprendizaje y reúnen a su alrededor diferentes materias, pues su carácter globalizador permite enhebrar los diferentes contenidos curriculares.

Por otra parte, "la construcción de una cultura educativa sólidamente sustentada en una práctica de investigación, pasa por identificar el capital cultural con el que cuenta la escuela como institución y espacio educativo, y generar los instrumentos de aprendizaje adecua- dos que permitan al personal docente aprender cómo investigar en sus propios espacios laborales, y a utilizar los resultados de sus investigaciones como base para la toma de decisiones en torno a su quehacer académico." (Alfaro 2007), cultura educativa, entorno educativo, instrumentos educativos, son el conjunto de requerimientos necesarios para una base sólida, que pueda propiciar el re direccionamiento las actividades investigativas, las cuales generen mejoras periódicas en términos educativos.

"la integración curricular favorece la educación inclusiva, evita la fragmentación de contenidos, da significado y funcionalidad a los aprendizajes, permite la sistematización de disciplinas para trabajar en proyectos integrados, evita el deterioro de la calidad académica, acerca la realidad a las aulas y hace conexiones entre el mundo real y el mundo de las aulas." (Molina 2011), buscar continuamente por todos los medios una educación de calidad permanente.

Es escasa la documentación que anteceda la integración de la investigación como estrategias pedagógica al currículo, pero si se encuentran estudios realizados sobre la transversalidad como eje en el currículo con fines de integrar estrategias que dinamicen el aprendizaje de los estudiantes, como es el caso: de la integración de las TIC al currículo, proyecto liderado por Illa E. López Jiménez y Camille Villafañe Rodríguez de la UNAD, cuyos resultado han tenido como impacto la implementación de las TIC en la práctica docente, de igual manera, los resultados de la Investigaciónaccionen la transformación e integración del currículo proyecto llevado a cabo en la I.E.D Delia Zapata Olivella, el cual será pilares de sustento en la presente investigación. 
Tabla 1

Contenidos del proyecto Educativo Institucional (PEI) y su articulación con la IEP.

\begin{abstract}
Definiones Conceptuales
Aprendizajes fundados en la Investigación.

Exponen cierto grupo de características fundamentales del concepto de aprendizajes fundados en la investigación, lo cual permite constituir el procedimiento concreto de acción y desarrollo de las prácticas educativas. Son los semilleros investigativos los que fomentan la cultura investigativa la adquisición de destrezas metodológicas, sociales y cognitivas que consienten el acercamiento y reconocimiento de las problemáticas sociales, planteando soluciones a ellas, a través de un método científico riguroso y sistemático y que (Echeverry, 2009; Martínez, 2017).
\end{abstract}

Investigación como estrategia pedagógica (IEP).

La IEP se fundamenta en el método científico, por tanto, es conveniente precisar el significado de Ciencia. De acuerdo con Palacio Salazar (2010), es la ciencia la que permite conocimiento sistematizado, elaborado mediante observaciones, razonamientos y pruebas metódicamente organizadas. Otro constructo teórico que alimenta a la I E. P. es el aprendizaje colaborativo, lo cual permite que se produzca una negociación cultural del conocimiento, de teorías, de modelos de organización que desemboca en un gran escenario que propicia el diálogo de saberes y la conformación sólida de comunidades que producen conocimiento, saber, práctica y están dispuestas a transformar la realidad. (Martínez, 2014; Guerrero 2016).

Práctica pedagógica e investigación.

Duque (2016), la investigación como estrategia pedagógica ejerce un impacto vinculante entre la práctica pedagógica y la investigación, se le brinda a las niñas y niños la posibilidad de apropiarse de la lógica del conocimiento y de herramientas propias de la ciencia con la mirada puesta en la sociedad y sus necesidades, proyectándonos al conocimiento significativo al que se refiere Ausubel (2001): para atribuir significado al material objeto de aprendizaje no sólo se deben actualizar sus esquemas de conocimiento sino también revisar, modificar y enriquecer este conocimiento.

\title{
Transversalidad.
}

Velásquez Sarria, J A; 2009) refiere lo transversal relacionándolo con dos conceptos: “cruzar” y “enhebrar". En el ámbito educativo, entonces, la transversalidad se refiere a una estrategia curricular mediante la cual algunos ejes o temas considerados prioritarios en la formación de nuestros estudiantes, permean todo el currículo, es decir, están presentes en todos los programas, proyectos, actividades y planes de estudio contemplados en el Proyecto Educativo Institucional-PEI- de una institución educativa. La transversalidad curricular implica como lo afirma Fernández (2003:5): "la utilización de nuevas estrategias, metodologías y necesariamente formas de organización de los contenidos".

Integración curricular de las TIC.

Es el proceso de hacerlas enteramente parte del currículum, permeándolas con los principios educativos y de la didáctica los cuales conforman el engranaje del aprender, a través de un uso armónico y funcional para un propósito de aprender específico. En este sentido, Sánchez (2002), citado en Abarzúa y Cerda (2011), establece que una adecuada integración de TIC implica utilizar transparentemente las tecnologías para facilitar la construcción del aprendizaje. Muchos de los modelos o estrategias existentes se basan en la organización de ambientes de trabajo colaborativo, apoyados por teorías constructivistas, donde las TIC son visualizadas como recursos de apoyo al trabajo docente y al aprendizaje de los alumnos. Un claro ejemplo corresponde a iniciativas de aprendizajes basados en proyectos que promueven el trabajo colaborativo de alumnos a través de la red (Campos y otros, 1998, citados en Abarzúa y Cerda, 2011).

Fuente Elaboración propia. (2018). 


\section{Metodología}

La investigación está orientada de acuerdo al paradigma Crítico - Social, porque se fundamenta en la reflexión crítica que busca la reforma de las instituciones que hacen parte de la realidad social a la que pertenecen los investigadores, en este caso los docentes.

La investigación es de tipo cualitativo, porque como lo señala Cerda (2003), tiene como objetivo la descripción de las cualidades de un fenómeno. El diseño de la investigación es transeccional porque se recolectan datos en un solo momento, en un tiempo único. Descriptivo, ya que describe la incidencia de factores en la situación que se está abordando.

Técnicas e instrumentos de recolección de la información

Para el caso de la presente investigación se empleó a los estudiantes una entrevista Estructurada y la observación, la cual fue registrada en un diario de campo.

\section{Escenario y actores}

El escenario donde se desarrolla el estudio es la Institución educativa de Ricaurte en donde se contó con la participación de 40 estudiantes de diferentes grados.

\section{Procedimiento}

Momento (1): Se seleccionan los instrumentos a utilizar.

Momento (2): Se especifican las categorías de estudio.
Momento (3): La entrevista estructurada se llevó a cabo de acuerdo a un proceso que contó con la participación de los docentes investigadores, lluvia de ideas, la redacción y la selección de preguntas, hasta la validación de la misma. Para este análisis se tuvo en cuenta la pregunta orientadora. ¿Cómo articular los lineamientos de la I.E.P. al currículo de la institución educativa rural María Auxiliadora?

Momento (4): Con base a las categorías de estudio: Radio Escolar, Herramientas pedagógicas, e Investigación; se aplicó a los participantes la entrevista, la cual pretende describir el fenómeno que se estudia partiendo del nivel de la investigación (no experimental) en donde se observa, y analiza tal y como acontece.

Momento (5): Una vez se tiene los resultados de la entrevista estructurada y la observación registrada se interpretan y comprenden los resultados a la luz de lo que exponen los teóricos que soportan la investigación.

Momento (6): Se relacionan los resultados y se muestran los hallazgos.

\section{Escenarios y Actores}

El escenario escogido para el presente estudio es la Institución Educativa de Ricaurte. Los actores fueron (40) estudiantes cuyas edades oscilan entre 10 y 15 años de edad.

\section{Resultados}

A continuación, se presentan los hallazgos derivados del instrumento aplicado, (entrevista estructurada). 


\begin{tabular}{|c|c|c|c|}
\hline $\begin{array}{l}\text { Categoría de } \\
\text { estudio }\end{array}$ & $\begin{array}{c}\text { Pregunta } \\
\text { orientadora }\end{array}$ & Discurso de los actores & Articulación y sistematización teórica \\
\hline $\begin{array}{l}\text { Herramientas } \\
\text { pedagógicas }\end{array}$ & $\begin{array}{l}\text { ¿Te gustaría } \\
\text { que tus } \\
\text { docentes } \\
\text { implementen } \\
\text { en clases } \\
\text { estrategias } \\
\text { de } \\
\text { investigación } \\
\text { con el apoyo } \\
\text { de las TIC?, } \\
\text { ¿Por qué? }\end{array}$ & $\begin{array}{l}\text { "Si porque tendríamos } \\
\text { una información } \\
\text { completa y segura", } \\
\text { "Si, con las TIC } \\
\text { aprendemos en } \\
\text { menos tiempo", } \\
\text { "Si, tendríamos } \\
\text { posibilidades de } \\
\text { aprender cosas } \\
\text { nuevas", "Si, nos } \\
\text { enseñarian a usar la } \\
\text { tecnología, "Si, me } \\
\text { gustaría demasiado, } \\
\text { las clases serian } \\
\text { más entretenidas", } \\
\text { "Si y no, porque } \\
\text { nos informariamos } \\
\text { más a fondo, pero } \\
\text { nos volveríamos } \\
\text { más flojos", "Si, la } \\
\text { tecnología es un apoyo } \\
\text { para el investigado" }\end{array}$ & $\begin{array}{l}\text { Ante las respuestas positivas frente a la } \\
\text { implementación de estrategias de investigación } \\
\text { con el apoyo de los tics en el aula de clases, pues } \\
\text { asumen que esto contribuiría significativamente } \\
\text { a mejorar la calidad educativa. Lo anterior } \\
\text { contribuye a lo que expresa Ciro (2012). Un } \\
\text { enfoque de enseñanza basado en proyectos se } \\
\text { construye sobre las fortalezas individuales de } \\
\text { los estudiantes y les permite explorar sus áreas } \\
\text { de interés dentro del marco de un currículo } \\
\text { establecido, además constituye un modelo de } \\
\text { instrucción auténtico en el que los estudiantes } \\
\text { planean, implementan y evalúan proyectos que } \\
\text { tienen aplicación en el mundo real más allá del } \\
\text { aula de clase. } \\
\text { Los hallazgos permiten observar que el } \\
\text { aprendizaje seria novedoso con la implementación } \\
\text { de herramientas tecnológicas, Así mismo es } \\
\text { posible inferir que una adecuada integración } \\
\text { de TIC implica utilizar transparentemente las } \\
\text { tecnologías para facilitar la construcción del } \\
\text { aprendizaje, ello como parte del currículum por } \\
\text { medio de una disciplina. ( Sánchez 2002, citado } \\
\text { en Abarzúa y Cerda, 2011), }\end{array}$ \\
\hline Investigación & $\begin{array}{l}\text { ¿Consideras } \\
\text { importante } \\
\text { que desde } \\
\text { tu IED se } \\
\text { generen } \\
\text { propuestas } \\
\text { de } \\
\text { investigación } \\
\text { para la } \\
\text { solución } \\
\text { de algún } \\
\text { problema } \\
\text { en tu } \\
\text { comunidad?, } \\
\text { ¿Por qué? }\end{array}$ & $\begin{array}{l}\text { "Si, se deben realizar } \\
\text { propuestas que } \\
\text { mejoren la calidad } \\
\text { de vida de nuestra } \\
\text { comunidad", "Si } \\
\text { porque el colegio } \\
\text { hace parte de la } \\
\text { comunidad", "Si, me } \\
\text { parece importante } \\
\text { que la sociedad } \\
\text { pueda apoyarse en la } \\
\text { IE", "Si, se apoya el } \\
\text { espiritu investigativo } \\
\text { y se solucionan los } \\
\text { problemas de la } \\
\text { comunidad", "Si } \\
\text { porque impulsaria } \\
\text { el desarrollo social } \\
\text { del pueblo", "Si } \\
\text { porque todo sería } \\
\text { más organizado y sin } \\
\text { tanto conflicto", "si } \\
\text { porque se ayudarian } \\
\text { mutuamente la } \\
\text { comunidad y la } \\
\text { institución" }\end{array}$ & $\begin{array}{l}\text { Lo hallazgos encontrados en los resultados de } \\
\text { la pregunta No } 2 \text { logran observar que todos los } \\
\text { estudiantes ven los proyectos de investigación } \\
\text { como una alterativa válida para darle respuesta } \\
\text { a los problemas de su comunidad, y esto trae } \\
\text { consigo la generación de nuevo conocimiento. De } \\
\text { acuerdo a lo expuesto resulta oportuno entonces, } \\
\text { acuñar el concepto de "Enseñanza Problémica", } \\
\text { también en las ciencias biológicas, entendido } \\
\text { como un sistema de métodos mediante el cual los } \\
\text { alumnos se enfrentan a ciertas contradicciones } \\
\text { de su objeto de estudio y las asimilan como } \\
\text { problemas, y exigen soluciones efectivas a través } \\
\text { de tareas y preguntas que contienen elementos de } \\
\text { problematicidad (Guanche, } 2005 \text { ). } \\
\text { Son los estudiantes los que ven el problema } \\
\text { como un cuestionamiento a lo que observan y } \\
\text { describen, dicha observación es constante, y es } \\
\text { donde predomina la capacidad para ver de qué } \\
\text { forma se convierten en curiosos científicos, para } \\
\text { dar respuesta a esas necesidades específicas. } \\
\text { Tal como lo expresa Mauffette, (2007), en estos } \\
\text { tiempos nos encontramos frente a un nuevo } \\
\text { tipo de estudiante, que llega con necesidades } \\
\text { y exigencias diferentes, y con habilidades y } \\
\text { competencias intelectuales definidas, que exigen } \\
\text { transformar los modelos de enseñanza y que } \\
\text { posibilitan aplicar metodologías basadas en la } \\
\text { construcción activa de significados conceptuales y } \\
\text { en la solución de problemas (Woods, 2006). }\end{array}$ \\
\hline
\end{tabular}




\begin{tabular}{|c|c|c|c|}
\hline Radio Escolar & $\begin{array}{l}\text { ¿Te gustaría } \\
\text { ser parte de } \\
\text { la solución } \\
\text { de algún } \\
\text { problema } \\
\text { propio de tu } \\
\text { comunidad } \\
\text { direccionado } \\
\text { por los } \\
\text { docentes de } \\
\text { tu IED? }\end{array}$ & $\begin{array}{l}\text { "Si, porque así } \\
\text { podría saber cómo se } \\
\text { soluciona un problema } \\
\text { de la comunidad, como } \\
\text { por ejemplo a través de } \\
\text { una radio escolar", "Si, } \\
\text { porque me considero } \\
\text { una persona muy } \\
\text { solidaria", "Si me } \\
\text { gustaría porque me } \\
\text { sentiría ayudada por } \\
\text { mis docentes, su apoyo } \\
\text { es muy importante", } \\
\text { "Si, no hay nada mejor } \\
\text { que la colaboración } \\
\text { para el bien común", } \\
\text { "Si, me gustaría que } \\
\text { apoyen mis ideas, y a } \\
\text { través de una radio } \\
\text { escolar seria fabuloso" }\end{array}$ & $\begin{array}{l}\text { Con base a la pregunta } \mathrm{N}^{\circ} 3 \text { se evidenciaron } \\
\text { hallazgos tales como; los estudiantes quieren } \\
\text { ser autores de soluciones de problemas propias } \\
\text { del aula de clases, recibiendo el apoyo de sus } \\
\text { docentes y la colaboración de sus compañeros. } \\
\text { Esto se fundamenta en lo que exponen los } \\
\text { autores Collazos y Mendoza, (2006). Sobre } \\
\text { aprendizaje colaborativo, definiéndola como un } \\
\text { área prominente para la investigación porque } \\
\text { les facilita a los aprendices razonar acerca de la } \\
\text { colaboración. Aunque para lograr un ambiente } \\
\text { efectivo para el aprendizaje colaborativo se debe } \\
\text { observar detenidamente la situación, observar } \\
\text { detenidamente la calidad de las interacciones. } \\
\text { Así mismo hablan de la asignación de roles como } \\
\text { una estrategia que logra potenciar ciertas áreas } \\
\text { específicas del saber, a través de la situación en } \\
\text { la cual se espera que ocurran formas particulares } \\
\text { de interacción que producirán mecanismos de } \\
\text { aprendizaje. (Roselli, 2011). }\end{array}$ \\
\hline $\begin{array}{l}\text { Herramientas } \\
\text { pedagógicas } \\
\text { Investigación } \\
\text { Radio Escolar }\end{array}$ & $\begin{array}{l}\text { ¿Alguna } \\
\text { vez en tus } \\
\text { experiencias } \\
\text { académicas } \\
\text { has hecho } \\
\text { uso de la } \\
\text { investigación } \\
\text { y las TIC? }\end{array}$ & $\begin{array}{l}\text { "Si", "Si he } \\
\text { investigado con mis } \\
\text { compañeros en Ciclón", } \\
\text { "Si, mis profesores } \\
\text { dicen que vamos a } \\
\text { hacer proyectos afuera } \\
\text { d la institución", } \\
\text { "Si y cada vez que } \\
\text { hacemos el proyecto } \\
\text { lo disfrutamos", } \\
\text { "Si las clases son } \\
\text { entretenidas y puedo } \\
\text { participar más", "Si } \\
\text { y quiero continuar } \\
\text { y que Ciclón siga } \\
\text { viniendo", "Si, Que las } \\
\text { clases sean siempre } \\
\text { así, que nos pongan } \\
\text { a hacer proyectos", } \\
\text { "No", "No pero quisiera } \\
\text { participar" }\end{array}$ & $\begin{array}{l}\text { Al respecto, Ciro (2012), señala que la propuesta } \\
\text { del trabajo por proyectos de investigación, } \\
\text { pretende brindar un apoyo en el ámbito educativo, } \\
\text { para la implementación de una metodología que } \\
\text { complemente los temas de las clases teóricas } \\
\text { con la aplicación de proyectos diseñados para } \\
\text { este fin y a la vez, incentivar mediante su } \\
\text { realización, actividades como: investigación, } \\
\text { planeación, búsqueda de soluciones, trabajo } \\
\text { cooperativo y actitudes como: autorregulación, } \\
\text { disciplina y perseverancia, entre otros elementos } \\
\text { que benefician la formación integral de los } \\
\text { estudiantes. } \\
\text { En tal sentido, la autora enfatiza en que trabajar } \\
\text { por proyectos no excluye la importancia de la } \\
\text { teoría, es decir, no significa perder el rigor de la } \\
\text { enseñanza, ya que los contenidos siguen siendo } \\
\text { la base del aprendizaje, solo que el secreto está } \\
\text { en hallar el punto de equilibrio entre teoría y } \\
\text { práctica. } \\
\text { Por tanto, es importante señalar que los } \\
\text { programas basados en la investigación no } \\
\text { deben perder el objetivo del acto pedagógico } \\
\text { de transformar conocimientos teóricos en } \\
\text { aprendizajes significativos. }\end{array}$ \\
\hline
\end{tabular}

Fuente: Elaboración propia (2018).

\section{Conclusión}

Los estudiantes ven la investigación como una de las herramientas primordiales que impactan en el quehacer educativo, y de esa manera se motivan por aprender a hacerlo a través de las necesidades que se presentan en su entorno. Y son las herramientas tecnológicas las que se han convertido en uno de los mejores elementos de innovación y transformación social. 
Son los maestros quienes motivan, propician, guían y retroalimentan en sus áreas específicas de conocimiento actividades que promuevan la curiosidad científica, la participación constante y los intereses específicos.

Asumen que el aprendizaje debe ser colaborativo, que las investigaciones no se realizan de manera aislada, y que la integración de conocimientos genera nuevas maneras de aprender, y además de convertir las necesidades colectivas en resultados participativos.

Finalmente han tenido la oportunidad de ser líderes de ideas en el aula de clase, y han sentido el respaldo de sus maestros, quienes facilitan ideas interactivas y de interconexión.

\section{Referencias}

Abarzua, A. y Cerda, C. (2011). Integración curricular de TIC en educación parvularia. Revista de Pedagogía, 32(90), 13-43. Recuperado de: http:/www.redalyc.org/articulo. oa?id=65920055002

Azorin, C. (2015). Integración pedagógica de wix en educación primaria. Pixel-Bit. Revista de Medios y Educación, 163-177. Recuperado de http://www.redalyc.org/articulo. oa?id=36841180011

Blanco, M. (2005). La educación de calidad para todos empieza en la primera infancia. Revista Enfoques Educacionales. 7(1): 11 - 33. Chile. Documento Recuperado de: http://www.facso. uchile.cl/publicaciones/enfoques/09/ Blanco-DelPiano_N7_2005.pdf

Castro, C. (2017) Valores Organizacionales en Institutos Universitarios. Revista REDHECS, $23 \mathrm{http://ojs.}$ urbe.edu/index.php/redhecs/issue/ view/68.
Ciro, C. (2012). Aprendizaje Basado en Proyectos (A.B.Pr) Como estrategia de enseñanza y Aprendizaje en la Educación Básica y Media. Universidad nacional de Colombia. Recuperado de: www.bdigital.unal.edu. co/9212/1/43253404.2013.pdf

Cuevas, A., Hernández, R., Leal, B., Mendoza, C. (2016). Enseñanza-aprendizaje de ciencia e investigación en educación básica en México. REDIE 18(3). Recuperado de: http://www.scielo.org.mx/scielo.php?script=sci_artte xt\&pid=S1607-40412016000300014

Duque, I. (2016). La investigación como estrategia pedagógica en el aula. Compartir aula maestra. Documento Recuperado de: https://compartirpalabramaestra.org/columnas/la-investigacion-como-estrategia-pedagogicaen-el-aula

Garzón, E. y Acuña, L. (2016). Integración de los proyectos transversales al currículo: una propuesta para enseñar ciudadanía en ciclo inicial. Revista Electrónica "Actualidades Investigativas en Educación”, 16. 1-26. Recuperado de http://imss.redalyc. org/articulo.oa?id=44746861010

Maranto, M. y González, M. (2015). Fuentes de Información. Universidad Autónoma del Estado de Hidalgo. Documento Recuperado de: http:// repository.uaeh.edu.mx/bitstream/ bitstream/handle/123456789/16700/ LECT132.pdf?sequence=1

Martinez, J. C., Berthel, Y., \& Vergara, M. S. (2017). Síndrome de Burnout en profesores y su relación con el aprendizaje de los estudiantes de básica primaria de una institución educativa oficial de Sincelejo (Colombia). Revista Salud Uninorte, 33 (2) .118-128. http://dx.doi.org/10.14482/ sun.33.2.10538 
Martínez, Royert J. C. (2014). Modelo de evaluación del Proceso de Atención de Enfermería. [Tesis doctoral], Universidad del Atlántico, Atlántico, Barranquilla. doi:10.13140/ RG.2.2.35792.00000

Mejía, M. \& Manjarrez, M. (2011). La investigación como estrategia pedagógica una apuesta por construir pedagogías críticas en el siglo xxi. Revista de educación y pedagogía Praxis y Saber.

Mejía, M. \& Manjarrez, M. (2013). La investigación como estrategia pedagógica una propuesta desde el sur. Ediciones desde abajo. Bogotá. Colombia.

Orjuela, D. (2010). Acercamiento a la integración curricular de las tic. Praxis \& Saber. 1. 111-136. Recuperado de http://www.redalyc.org/articulo. oa?id $=477248386007$
Salcedo, J. (2009). La investigación Acción en la transformación e integración curricular. Un diálogo crítico desde la IED Delia Zapata Olivella. Revista actualidad pedagógica 54. Recuperado de: https://revistas.lasalle.edu.co/index.php/ap/article/download/961/868

Sánchez, H. (2013). La actividad científico - investigativa en educación. Revista Científico-Metodológica, 57, 21-25, julio-diciembre. Recuperado de: http/:www.redalyc.org/articulo. oa?id=360634164005

Villarroel, F. (2012). El constructivismo. y su papel en la innovación educativa. Revista de Educación y Desarrollo, 20. Recuperado de: www.cucs.udg. $\mathrm{mx} / \mathrm{revistas} / \mathrm{edu}$ _desarrollo/anteriores/20/020_Villarruel.pdf 